... René Prêtre, Chefarzt Herzchirurgie am Zürcher Kinderspital, Professor an der Universität Zürich - und «Schweizer des Jahres 2009»

\title{
«Im Zentrum von allem, immer das Herz ...»
}

\section{Daniel Lüthi}

Text und Bilder

* in diesem Fall ein ECMO (extracorporeal membrane oxygenator), der den Kreislauf einige Wochen lang unterstützen kann

danielluethi@gmx.ch
Welch verschiedene Welten, die da ganz nahe beieinanderliegen! In der Ecke der alten Herrschaftsvilla: das Büro. Wie ein Wohnzimmer wirkt es, gemütlich fast dank einer wohltuenden Unordnung, im Büchergestell Bildbände über bildende Künstler, an der Wand Fähnlein des Weltfussballverbandes FIFA, die hier auch hängen, wenn nicht Weltmeisterschaft ist, dazu zwei grossformatige Fotos mit Pelé an der WM 1970 in Mexiko. «Für mich die schönste WM aller Zeiten», schwärmt der feingliedrige Mann vis-à-vis, der seinen weissen Arztkittel inzwischen abgelegt hat. «Das war Pelé nach seinem wunderbaren Kopftor im Final gegen Italien, unglaublich, die Flugbahn des Balles - und schön auch der Respekt, den die Spieler damals noch füreinander hatten.»

Auf dem Pult zahlreiche Holzstatuen aus Afrika, einige Fussbälle - darunter auch der offizielle aus Süd- afrika, ja, überreicht von Sepp Blatter persönlich - ein altertümlicher Radioapparat, viel Papier und ein kleiner Laptop. Vor der grossen Fensterfront Terrasse und Garten. Und in Blickdistanz, in nur wenigen Schritten intern erreichbar: der moderne Trakt mit dem Hightech-Operationssaal, wo René Prêtre wohl die meiste Zeit seiner Tage verbringt, bei schwierigen Operationen sind es acht Stunden am Stück.

\section{Lebensretter - hier und dort}

Auf der Intensivstation, umgeben von Maschinen und Schläuchen, liegt ein 11 Monate altes Mädchen. René Prêtre lächelt leise: «Ja, das war knapp.» Akute Myokarditis, schwere Mitralklappen-Insuffizienz, extrem reduzierte Herzfunktion. Prêtre und sein Team setzten ein Kunstherz* ein, fünf Tage später konnten sie dieses wieder entfernen, bald wird die kleine Patientin auf

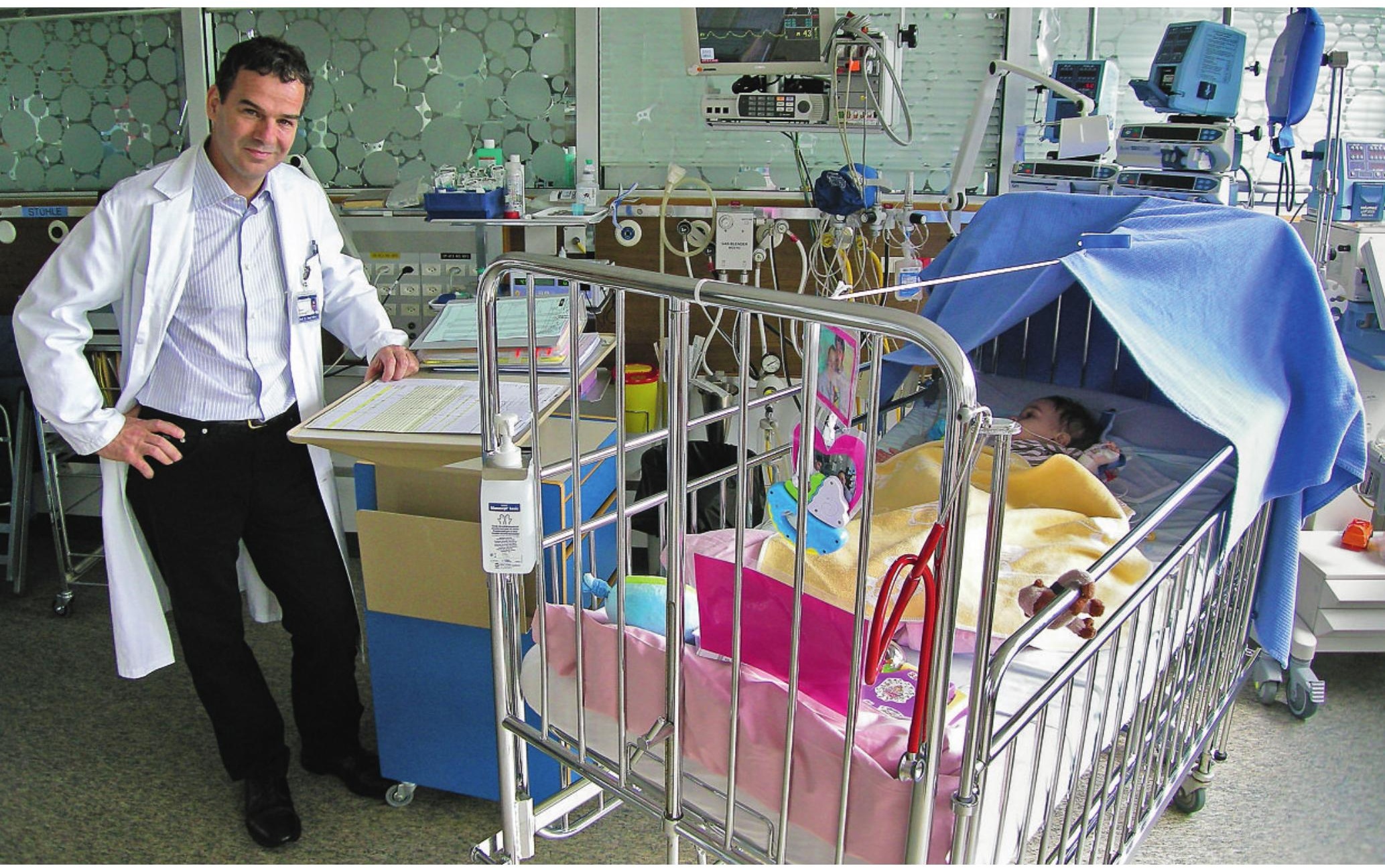


die Station verlegt werden können. «Mit grosser Wahrscheinlichkeit wird sie ein normales Kind», sagt Prêtre mit verhaltenem Stolz, «vor zehn Jahren wäre sie gestorben.» Ein Lebensretter, immer wieder, immer noch mit grossem Engagement, und immer noch mit berührender Bescheidenheit, was alles andere als selbstverständlich ist. Denn Prêtre ist ein Promi. Dies bezeugen die beiden Titel, die dieser Ausnahme-Arzt im Januar dieses Jahres anlässlich einer vom Fernsehen übertragenen, glamourösen Feier erhalten hat: Er wurde zum Schweizer des Jahres 2009 gewählt und damit in eine Reihe gestellt mit Bundesrätin Eveline Widmer-Schlumpf, Schwingerkönig Jörg Abderhalden oder dem ehemaligen Coach der Schweizer FussballNationalmannschaft, Köbi Kuhn. Prêtre weilte damals gerade in Afrika und wurde auf der Grossleinwand mit einer Video-Botschaft zugeschaltet. Typisch: Die Arbeit geht vor.

\section{Seine Bescheidenheit ist beeindruckend - die Tatsache aber bleibt: Prêtre gilt als Star-Chirurg}

Trotzdem: Prêtre ist einer der wenigen Chirurgen, den man (und frau) auf der Strasse erkennt und grüsst, weil er via Fernsehen oder Schweizer Illustrierte bei Herrn und Frau Schweizer auch schon im Wohnzimmer weilte. Das Publikum verlieh ihm im Januar denn auch noch den Titel «Swiss Award» in der Kategorie «Gesellschaft», wohl vor allem wegen seines regelmässigen Engagements für die Stiftung «Le petit Cœur» in Mosambik. Der «König der Herzen», der in Afrika für Gottes Lohn arme schwarze Kinder heilt: Das ist der Stoff, aus dem die Arztromane sind. Prêtre relativiert, mit Nachdruck: «Das ist nicht mein Projekt, ich habe es nicht aufgebaut, sondern bin bloss beteiligt. Auch arbeite ich pro Jahr ja nur zwei Wochen lang in Maputo. Nein, nein, ich bin nicht Beat Richner, die Entwicklungshilfe ist nicht mein Leben, meine Arbeit ist hier in Zürich, am Kinder- und am Unispital, hier operiere, forsche und lehre ich, das ist meine Priorität, und das ist auch der Hauptgrund für meine Auszeichnungen.»

In Maputo operieren Prêtre und sein Schweizer Team mit rund acht Leuten nicht die ganz schwierigen Fälle. In der Regel ist weder eine Nachbetreuung der Patienten noch eine Kommunikation mit deren Eltern möglich, hier überwiegt bei den Operationen also die technische Seite. Die menschliche, die in der Schweiz oft zu kurz komme, funktioniere in Afrika dafür beim Team besser, vor allem nach der Operation: Gemeinsam gehe man Spaghetti essen und erzähle sich Geschichten aus dem Leben.

\section{Handwerker und Künstler}

Wenn Prêtre erzählt, spricht er leise. Und wenn die Rede auf einen seiner zahlreichen Erfolge kommt, die

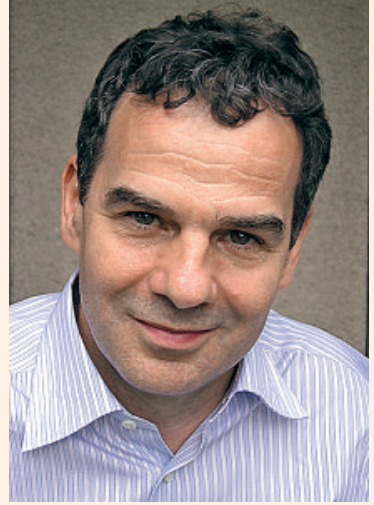

\section{René Prêtre}

Prof. Dr. med. René Prêtre ist Herzchirurg und Spezialist für Kinder-Herzchirurgie. Seit 2001 ist er Chefarzt Chirurgie am Zürcher Kinderspital, an der Universität Zürich ist er Professor. Im Januar dieses Jahres wurde Prêtre zum «Schweizer des Jahres 2009» gewählt. Gleichzeitig erhielt er den «Swiss Award 2009» in der Kategorie «Gesellschaft». Geehrt wurde er mit diesen prestigeträchtigen Titeln unter anderem auch für sein Engagement in der Stiftung «Le Petit Cœur» in Mosambik. Dort arbeitet er mit seinem Team ehrenamtlich während zwei Wochen pro Jahr.

René Prêtre wurde 1957 als drittältestes von sieben Kindern in Boncourt im Jura geboren. Nach dem Medizinstudium in Genf - mit dem Staatsexamen 1982 - zog er nach New York, wo er vor allem Opfer von Gewaltverbrechen und Unfällen operierte. 1994 wirkte er am Great Ormond Hospital in London, im Jahr 2000 am Hôpital Necker in Paris, immer als Herzchirurg.

Prêtre ist geschieden und Vater von zwei erwachsenen Töchtern. Er lebt in Zürich.

ihn auch international zu einer Koryphäe gemacht haben, senkt er, verschämt fast, den Blick. Auch jetzt, wo wir darüber sprechen, wie sein Name vor kurzem neben jenen der sieben bisherigen Schweizerinnen und Schweizern des Jahres in den Gedenkstein auf der Älggi-Alp im Kanton Obwalden eingemeisselt wurde, dem geografischen Mittelpunkt der Schweiz.

Seine Bescheidenheit ist beeindruckend - die Tatsache aber bleibt: Prêtre gilt als Star-Chirurg. Seine Reaktion auf dieses Etikett ist erfrischend: «Ich bin kein Star-Chirurg, ich habe noch nie einen Stern operiert. Ich bin Herz-Chirurg.» Mit Haut und Haar ist er das vor allem aber: mit ganzem Herzen. Und dies ist für ihn weit mehr als ein Organ. «Natürlich ist das Herz auch ein Muskel und eine Pumpe. Vor allem aber ist das Herz ein faszinierender Körper, der lebt - und sein 
Leben, im Gegensatz zu einer Leber beispielsweise, auch zeigen kann. Das ist Physiologie und Anatomie, aber auch Rhythmus. Es ist jedes Mal wie ein Wunder, wenn ein Herz nach einer Stunde Stillstand wieder zu schlagen beginnt, wenn die Zellen erwachen.» Wieder leuchten die Augen von Prêtre, wieder brennt das Feuer der Faszination, wieder ist der Enthusiasmus fast so greifbar wie das Herz-Modell oder der grosse Bergkristall auf dem Tisch. Dort liegt auch ein Buch, an dem er mitgearbeitet hat. Auf der ersten Seite die Widmung: «Im Zentrum von allem, immer das Herz ...»

Die Dreidimensionalität sei es unter anderem, die ihn begeistere, fährt Prêtre fort, steht auf und zeigt auf ein paar Bücher im Gestell. Alberto Giacometti, Aristide Maillol, Michelangelo, Rodin - Bildhauer gefallen ihm. «Oder schauen Sie hier, diese wunderbaren Skulpturen von Picasso: dieser Hund, diese Katze, dieser Mann mit dem Schaf, dieses Gleichgewicht.» Und wieder werden Parallelen augenfällig: Prêtre zeichnet und hat mit Ton modelliert. Und auch im Operationssaal fühlt er sich gleichzeitig als Handwerker und Künstler, konstruiert und rekonstruiert. «Ich will immer sehr präzis arbeiten, aber auch elegant, ästhetisch, schön. Möglichst wenig invasiv und nie brutal, mit Respekt für das Gewebe, beispielsweise.»

\section{René Prêtre ist nicht Einzelkämpfer, sondern Teamplayer. Im Spital und im Sport}

\section{Faszination Fussball}

René Prêtre ist nicht Einzelkämpfer, sondern Teamplayer. Im Spital und im Sport. «Joggen oder schwimmen ist nichts für mich, das ist mir zu einsam, lieber spiele ich Ping-Pong mit jemandem, oder ein Mannschaftsspiel.» Wir sind wieder beim Fussball, die Augen des Gegenübers leuchten. Liebevoll blättert Prêtre in einem Heft mit kopierten Zeitungsartikeln und schwelgt in Erinnerungen: «Sehen Sie, das bin ich mit meinem Kopfballtor, 1977 etwa, mit dem FC Boncourt gewannen wir damals 3:0 gegen Köniz. Da spielten wir gegen Binningen - und hier, gegen Langnau, verloren wir.» Faszination Fussball: Was macht sie aus? «Gemeinsam kämpft man, gemeinsam gewinnt oder verliert man - das sind Emotionen, das ist wie ein Hollywood-Drama, nur lebendiger als im Kino, das ist wie ein Mikro-Trauma, wunderbar.» Das ist, und dieses
Bild wird immer deutlicher, wie im Operationssaal. Auch hier ist ein gutes Gefühl ebenso wichtig wie eine möglichst perfekte Technik, auch hier erfordert der Erfolg viel Training, auch hier braucht es Persönlichkeiten, und auch hier ist Teamgeist gefragt: «Kardiologen, Intensivmediziner und wir Herzchirurgen arbeiten quasi im Dreieck, nur gemeinsam sind wir stark, gemeinsam kämpfen wir ... ja, auch gegen den Tod.» Manchmal, sehr selten, geht etwas schief, macht auch ein sogenannter «Star-Chirurg» einen Fehler. «Es gibt ein Recht, Fehler zu machen, gerade zu Beginn einer Karriere. Davon profitieren dann später vielleicht andere.» Ja, er selber habe eine Situation auch schon falsch eingeschätzt, habe als Folge davon auch schon ans Aufhören gedacht. «Es gibt Eltern, die zu Recht böse auf mich sein dürfen. Gleichzeitig aber frage ich mich in Momenten des Zweifels immer auch: Wie viele Kinder leben dank uns?» 350 Operationen etwa führen Prêtre und seine Teams pro Jahr durch, 280 davon an Kinderherzen. Nicht immer und nicht zu jedem Preis wird operiert, bei stark einschränkenden Mehrfach-Behinderungen zum Beispiel, wenn keine Aussicht besteht, die Lebensqualität eines kleinen Patienten zu verbessern, entscheidet sich das verantwortliche Team gegen einen Eingriff. «Die Natur produziert auch Fehler, und manchmal können wir dies nicht beeinflussen, müssen der Natur ihren Lauf lassen, ohne schlechtes Gewissen.»

\section{Welten wachsen zusammen}

Übrigens: Prêtres Einstieg in den Beruf war eher Zufall als Berufung: «Eigentlich hätte ich Landwirt werden wollen, hätte ich den Bauernhof meiner Eltern übernehmen sollen. Aus ökonomischen Gründen war dies damals jedoch noch nicht möglich, deshalb schrieb ich mich, eine Stunde vor Ablauf der Frist, an drei Fakultäten der Uni ein. Ich entschied mich dann für Medizin, weil ich spürte, dass sie mir das ganze Spektrum offeriert.»

Das ganze Spektrum. Verschiedenste Facetten, die ein Ganzes ergeben: Landwirtschaft und Herzchirurgie, bildende Kunst und Sport, der Star und der bescheidene Mensch, der arrivierte Chefarzt im Spital und der bubenhafte Fussball-Fan am Spielfeld - das gemütliche Büro in der alten Villa und der sterile Operationssaal im modernen Trakt nebenan.

Am Ende einer langen, tiefschürfenden und nachhaltigen Begegnung sind Welten, die zu Beginn noch so verschieden wirkten, ganz organisch zu einer Einheit zusammengewachsen.

\section{Die nächste «Begegnung mit ...»}

Am Ende jeden Monats stellt die Schweizerische Ärztezeitung eine Persönlichkeit vor, die sich im Gesundheitswesen engagiert. Im August schildert Daniel Lüthi seine Begegnung mit François Mottu, Allgemeinmediziner, Familienarzt in Versoix, Reisender, Tier- und Landschaftsfotograf. 